\title{
IMPACTO DEL CAMBIO CLIMÁTICO EN LA SALUD EN EL SALVADOR
}

\author{
Elmer Jehovani Portillo-Sorto ${ }^{1}$ \\ Recibido: 25/09/19 \\ Aceptado 02/01/20
}

\section{RESUMEN}

El cambio climático ocasiona fenómenos atmosféricos extremos, exceso de contaminación, aumento de plagas, aumenta la mortalidad por temperaturas extremas, escasez de alimentos y agua, efectos en plantas y animales, con implicaciones directas e indirectas en la salud. El objetivo es reflexionar y crear conciencia del impacto que tiene el cambio climático en la salud y, de esta manera, incentivar el inicio de acciones de prevención y promoción que ayuden a contrarrestar dicho impacto. La metodología consistió en la revisión bibliográfica y análisis descriptivo y transversal sobre el impacto del cambio climático en la salud en El Salvador. En conclusión, la problemática sobre el impacto del cambio climático en la salud debe ser abordada en forma conjunta con el involucramiento de sectores claves en estos procesos, como instituciones educativas públicas y privadas (escuelas, colegios, universidades), empresas, industrias, maquilas que fomenten una conducta proambiental, a fin de conservar y proteger el entorno, no sobrexplotar los recursos, disminuir el consumo de energía eléctrica, evitar la contaminación; además, conectar nuestras actividades equilibradamente con los procesos ambientales en curso. Así mismo, impulsar, profundizar y defender una genuina y amplia participación social y comunitaria en salud y medioambiente, asegurar mecanismos de financiamiento y de asignación de recursos que sean solidarios y eficientes; realizar investigación y evaluación del impacto en salud del cambio climático en El Salvador.

Palabras clave: cambio climático, calentamiento global, salud, El Salvador.

1/ Maestro en Salud Pública, Doctor en Cirugía Dental, Docente en Universidad Evangélica de El Salvador Jehovaniportillo@icloud.com 


\title{
CLIMATIC CHANCE IMPACT ON HEALTH, EL SALVADOR
}

\begin{abstract}
Introduction: The climatic change causes extreme atmospheric phenomena, excess of contamination, plague increase, mortality increase due to extreme temperatures, lack of food and water, effects on plants and animals, with direct and indirect implications on health. Objective: To reflect and create conscience of the impact that the climatic change has on health, and in this way, encourage people to take actions of prevention and promotion that could help to counter the so called impact. Methodology: Bibliographic review and descriptive and transversal analysis on the impact of climatic change on health in El Salvador. Conclusion: The problems on the impact of climatic change on health, must be aboard together with the involvement of key sectors in these processes, such as public and private educative institutions (schools and universities), companies (or enterprises), industries, assembly plants, among others. Promoting a pro-environmental behavior, in order to conserve and protect the environment, not overexploit resources, reduce the consumption of electricity, avoid pollution; In addition, connect our activities in a balanced way with the environmental processes under way. Likewise, promote, deepen and defend a genuine and broad social and community participation in health and environment, ensure funding mechanisms and allocation of resources that are supportive and efficient; conduct research and evaluation of the health impact of climatic change in El Salvador.
\end{abstract}

Key words: Climatic change, global heating, health, El Salvador.

\section{Introducción}

Uno de los temas más discutidos en la actualidad es el cambio climático, considerado como el problema medioambiental más importante por su carácter global, persistencia, rapidez con que está ocurriendo y por la incertidumbre que conlleva.

El cambio climático se puede definir como un cambio de clima atribuido directa o indirectamente a la actividad humana que altera la composición de la atmósfera mundial y que se suma a la variabilidad natural del clima observada durante períodos comparables.

Desde tiempos antiguos la tierra ha experimentado fluctuaciones en el clima por temperatura, hu- medad, presión atmosférica, precipitaciones, entre otros fenómenos. En el transcurso del tiempo esto ha ido en aumento, actualmente esos cambios no solo obedecen a un fenómeno natural, sino que se le suma la intervención humana (tala de bosques, degradación de tierras y desertificación, agotamiento y contaminación del agua dulce, crecimiento poblacional y la urbanización) que puede llegar a inducir el Cambio Climático por medio de la intensificación del Efecto Invernadero a este aspecto se le conoce como «cambio climático antropogénico».

El cambio climático es, en parte, producto del incremento de las emisiones de los gases de Efecto 
Invernadero y denota un proceso que no es temporal y puede verificarse en el tiempo revisando datos climáticos, así mismo, es una alteración radical y brusca de los equilibrios medioambientales entre el hombre y la naturaleza.

El cambio climático es la pauta por excelencia de la crisis ambiental en la actualidad, generada especialmente por «la actividad de grandes empresas agroindustriales, proyectos de minería e hidroeléctrica en diversas comunidades que se ven impulsadas por intereses políticos y sociales particulares, dejando de lado las posibles y graves repercusiones en el medio ambiente». (Gunther, M.2014)

El cambio climático significa la alteración de los sistemas ecológicos y biofísicos de la tierra que se manifiestan por los cambios en la capa de ozono, pérdida de biodiversidad, daños en la producción alimentaria terrestre y marina, disminución de las fuentes de agua potable, y aumento de la contaminación ambiental (IPCC, 1996). (Woodward A, et al, 2000)

En este sentido, uno de los problemas con respecto al Cambio Climático es la incertidumbre en cuanto a las consecuencias o el impacto que produce. El cambio climático impacta directa o indirectamente la salud, debido a los efectos que produce en la naturaleza como sequías e inundaciones, retroceso en los glaciares, subida del nivel del mar, pérdida de zonas costeras, alteraciones de los ciclos del agua, aumento de la frecuencia de huracanes y otros elementos químicos y fenómenos atmosféricos extremos, exceso de contaminación, aumento de plagas, aumenta la mortalidad por temperaturas extremas, escasez de alimentos y agua, efectos en plantas y animales.

Lamentablemente los efectos del cambio climático producen una mayor pobreza en países que se encontraban en malas condiciones sociales y económicas, lo que consecuentemente lleva a más problemas sanitarios.
Así se han generado diferentes propuestas a nivel mundial para contrarrestar los efectos del cambio climático entre ellas las «basadas en energías alternativas a la generada por los combustibles fósiles, afirmando que solamente con políticas públicas enfocadas a la participación de las masas en la construcción consciente de una energética solar sustentable, se salvará la humanidad». (Martínez, J. T., 2013)

Otra de las propuestas es «la biotecnología vegetal que ayudará a reducir el tiempo y el costo de la producción, además, de estabilizar los ecosistemas de las zonas forestales, dándoles mayor oportunidad de regenerarse y así aumentar el carbono que requieren». (Álvarez Brito, 2013)

Por otro lado, se plantea especialmente «revertir los impactos sociales y culturales que implica la aplicación de determinados modelos de desarrollo que priorizando en la noción de crecimiento y en la generación de riqueza material, acaban siendo ambientalmente inviables, socialmente desiguales y culturalmente restrictivos». (De la Cuadra, Fernando, 2013)

\section{Cambio climático y su impacto en la salud}

Según la Organización Mundial de la Salud (OMS, 1948) «salud no es sólo la ausencia de enfermedad, sino también es el estado de bienestar somático, psicológico y social del individuo y de la colectividad». Es una definición progresista, en el sentido que considera la salud no sólo como un fenómeno somático (biológico) y psicológico, sino también social. La definición reconoce, acentúa y subraya que las intervenciones salubristas incluyen no sólo los servicios clínicos que se centran en su mayor parte en lo somático y psicológico, sino también las intervenciones sociales tales como las de producción, distribución de la renta, consumo, vivienda, trabajo y ambiente.

Se puede decir que la salud es un derecho humano y al mismo tiempo una necesidad, en la que interactúan factores orgánicos e inorgánicos, socia- 
les y ecológicos, es decir, intervienen elementos internos y externos del individuo, para el caso el cambio climático puede causar impacto en la salud.

\section{El impacto del cambio climático en la salud incluye:}

- Aumento de los efectos en la salud por causa de los eventos climáticos extremos (olas de calor y de frío, inundaciones, sequías, fuegos, etc.).

- Incremento de la frecuencia de las enfermedades respiratorias debido a los cambios en la calidad del aire y la distribución del polen.

- Aumento de la incidencia de las enfermedades de origen alimentario y transmitido por el agua, particularmente, en las áreas donde los sistemas de saneamiento son precarios.

- Cambio en la distribución de las enfermedades infecciosas o de sus vectores y contribución al posible establecimiento de especies tropicales o subtropicales.

¿Cuáles son los principales efectos directos del cambio climático sobre la salud que han sido detectados?

- Efectos cutáneos: Melanoma maligno, cáncer de piel no melanocítico (carcinoma basocelular, carcinoma escamocelular), quemaduras solares, dermatosis solar crónica, fotodermatitis.

- Efectos oculares: Queratitis y conjuntivitis actínica aguda, degeneración esferoidal de la córnea, pterigión, cáncer de córnea y conjuntiva, opacidad del cristalino (catarata): cortical, subcapsular posterior, melanoma uveal, retinopatía actínica aguda, degeneración macular.

- Efectos sobre la inmunidad y las infecciones: Supresión de la inmunidad celular, mayor susceptibilidad a las infecciones, menor eficacia de la inmunización preventiva, activación de infecciones víricas latentes.

El impacto del Cambio Climático, no solo afecta el clima, sino el Medio Ambiente y al ser humano; así, por ejemplo, los efectos de la sequía que son escasez de agua, dificultad de higiene, inseguridad alimentaria y contaminación del aire; traen consecuencias a la salud, manifestándose en enfermedades como asma, alergias, infecciones respira- torias agudas y dengue. Por otro lado, las lluvias provocan inundaciones, contaminación del agua e incremento de criaderos; afectando la salud con enfermedades diarreicas agudas, dengue, malaria y cólera.

El cambio climático conlleva fenómenos como «ciclones, huracanes, sequías, lluvias y enormes inundaciones» (Espejel A, y Flores A. 2015) que tienen un lamentable impacto en pérdida de vidas humanas; sin embargo, el efecto es mayor todavía porque estos fenómenos originan desastres que «inciden en los sistemas productivos, en las cosechas y en la agricultura, prolongando las consecuencias de estas turbulencias climáticas generándose así, la falta de alimentos, hambre, pobreza y diferentes flagelos que se mantienen en el tiempo». (Rozas O, 2003)

Los efectos del cambio climático en la salud pueden ser tanto directos como el aumento de morbimortalidad en relación con la temperatura; o indirectos, como las enfermedades transmitidas por insectos y enfermedades relacionadas con dificultades de acceso al agua potable y alimentos (Ochoa, $\mathrm{M}$, et al 2015). Además, el cambio climático favorece las condiciones ideales para la propagación de las infecciones y epidemias.

Según los informes del Panel Intergubernamental para el Cambio Climático y Sutherst, muestran que el cambio climático incrementa el número de casos y la presencia estacional de enfermedades como el dengue y paludismo. (IPCC 2001), (Sutherst R. 2001). Además, Kuhn K, y colaboradores coinciden en que las enfermedades infecciosas más frecuentes, en especial las transmitidas por vectores, son altamente sensibles a las variaciones climáticas. (Kuhn K, et al 2005)

Las temperaturas más altas aceleran el metabolismo de los insectos, incrementan la producción de huevos, la humedad crea un hábitat favorable para su desarrollo, las inundaciones al igual que la deforestación favorecen el desplazamiento de los insectos a zonas habitadas por el hombre. (Patz J.A, Kovats R.S. 2002) 
Como ya se mencionó, anteriormente, cuando se dan alteraciones en el clima, también la salud se ve comprometida, en forma directa o indirecta (Figura 1).

Además, es evidente la aparición de «trastornos psiquiátricos en las personas sanas por estrés postraumático, depresión severa y aparición de nuevas enfermedades infecciosas graves que impactan psicológicamente a las personas, debido al aumento de los desastres naturales» (González, et al, 2013). En este sentido, se debe considerar que la salud mental resulta afectada por los efectos del cambio climático por lo que se debe prevenir o contrarrestar estas repercusiones.

La salud humana es afectada por la contaminación del aire estrechamente relacionada con el cambio climático, que al mismo tiempo potencia el efecto de está, ambos originados en el modelo energético, es decir, son provocados por la actividad humana, su impacto en la salud es de largo alcance el cual es provocado por unos y lo sufren otros.

Ballester menciona que los «componentes relacionados con el clima, jugaban un papel modificador del efecto de la contaminación con la mortalidad. El efecto de las partículas sobre la mortalidad fue mayor en las ciudades de clima más cálido (Ballester, Ferran. 2005). Esto influye en el desarrollo y formación de las «enfermedades infecciosas y las enfermedades no infecciosas como: el asma, la rinitis alérgica y el eczema atópico» (Ochoa, M, et al 2015), entre otros. No se puede negar el hecho que un alto porcentaje de la población mundial está expuesta a la contaminación ambiental y, por ende, las consecuencias negativas para la salud van en aumento.

Según la Organización Mundial de la Salud (OMS), nueve de cada diez personas en el mundo respiran aire con altos niveles de contaminación y siete millones de ellas mueren cada año por la contaminación ambiental y doméstica. En el año 2016, la contaminación del aire provocó aproximadamente 4,2 millones de muertes, mientras que la contaminación del aire doméstico origi- nada por la cocción de alimentos con combustibles y tecnologías contaminantes causó unos 3,8 millones de muertes en el mismo periodo (WHO Household energy database, 2017).

Además, la OMS afirma que la contaminación del aire es un factor de riesgo crítico para las enfermedades no transmisibles (ENT), ya que se estima que causa una cuarta parte (24\%) de todas las muertes de adultos por cardiopatías, el $25 \%$ de las muertes por accidentes cerebrovasculares, el $43 \%$ de las muertes por neumopatía obstructiva crónica y el $29 \%$ de las muertes por cáncer de pulmón. Si bien es cierto que esto es una amenaza para todos, las personas más afectas son los pobres y marginados, en su mayoría mujeres y niños (WHO Household energy database. 2017).

Asimismo, tal como lo afirma la OMS, el cambio climático influye en los «determinantes sociales y medioambientales de la salud, a saber, un aire limpio, agua potable, alimentos suficientes y una vivienda segura». Según se prevé entre 2030 y 2050 el cambio climático causará «unas 250.000 defunciones adicionales cada año, debido a la malnutrición, el paludismo, la diarrea y el estrés calórico». (OMS, 2016)

Las temperaturas altas pueden provocar calambres, deshidratación, lipotimias, arritmias y exacerbación de enfermedades respiratorias y circulatorias ya existentes; especialmente en adultos mayores, en personas con problemas renales o con obesidad y en los que consumen drogas, alcohol o con determinada medicación (Kunst A.E, et al 1993), (Kenney W.L, 1987). Además, Las altas temperaturas pueden agravar los problemas cardiovasculares, debido a que la contaminación en el aire y los niveles de ozono aumentan.

¿Cuáles son las condiciones que determinan el impacto del cambio climático en la salud?

Según los informes del Panel Intergubernamental para el Cambio Climático existen tres condiciones. (IPCC 2001) 


\section{Condiciones individuales}

- Personas con peor salud: los que padecen enfermedades cardiovasculares, respiratorias o renales son más vulnerables a los efectos directos de las olas de calor o de la contaminación atmosférica; aquellos individuos con inmunidad comprometida pueden sufrir más en situaciones de temperaturas extremas o pueden contraer enfermedades infecciosas.

- Los ancianos corren más riesgo de padecer enfermedades infecciosas, efectos causados por temperaturas extremas y presentan, en general, peores condiciones físicas o, incluso, menor capacidad de adaptación o respuesta.

- Los niños presentan un mayor riesgo de enfermedad o muerte debido a la falta de madurez de algunos sistemas, a su mayor actividad y su menor tamaño; por ello corren más riesgo de diarreas, enfermedades ligadas a vectores y efectos directos del calor y de la contaminación atmosférica.

- Grupos con menores ingresos: la pobreza aumenta el riesgo porque tiene menos acceso a sistemas adecuados de acondicionamiento de aire, se vive en zonas urbanas más calurosas, se posee menos información para evitar exposiciones a situaciones extremas.

\section{Condiciones comunitarias}

La existencia de sistemas de abastecimiento de agua, de distribución de alimentos, de sistemas de alerta y de servicios de salud pública.

\section{Condiciones geográficas}

Poblaciones en zonas costeras bajas, poblaciones en los límites de las enfermedades transmitidas por vectores, poblaciones rurales alejadas de asistencia sanitaria, poblaciones urbanas sometidas al efecto de isla térmica.
¿Con que instrumentos cuenta el Salvador para enfrentar los efectos del cambio climático?

En el 2012, el Consejo de Ministros aprobó la Política Nacional del Medio Ambiente, también se realizaron reformas a la Ley del Medio Ambiente (LMA), incorporando el cambio climático en su estructura, específicamente se creó el Título VI-Bis que establece el fundamento para la elaboración del Plan Nacional de Cambio Climático y en el 2013 se elaboró la Estrategia Nacional del Medio Ambiente, que integra la Estrategia Nacional de Cambio Climático.

En el 2015 se presenta el primer Plan Nacional de Cambio Climático (PNCC), que consta de ocho componentes. Los sectores que contribuyeron en la confromación de dicho plan son: agropecuario, construcción, industrial, académico, municipal, líderes comunitarios, indígenas, no gubernamental, gubernamental, cooperación internacional, gremiales técnicas y consultores independientes. 


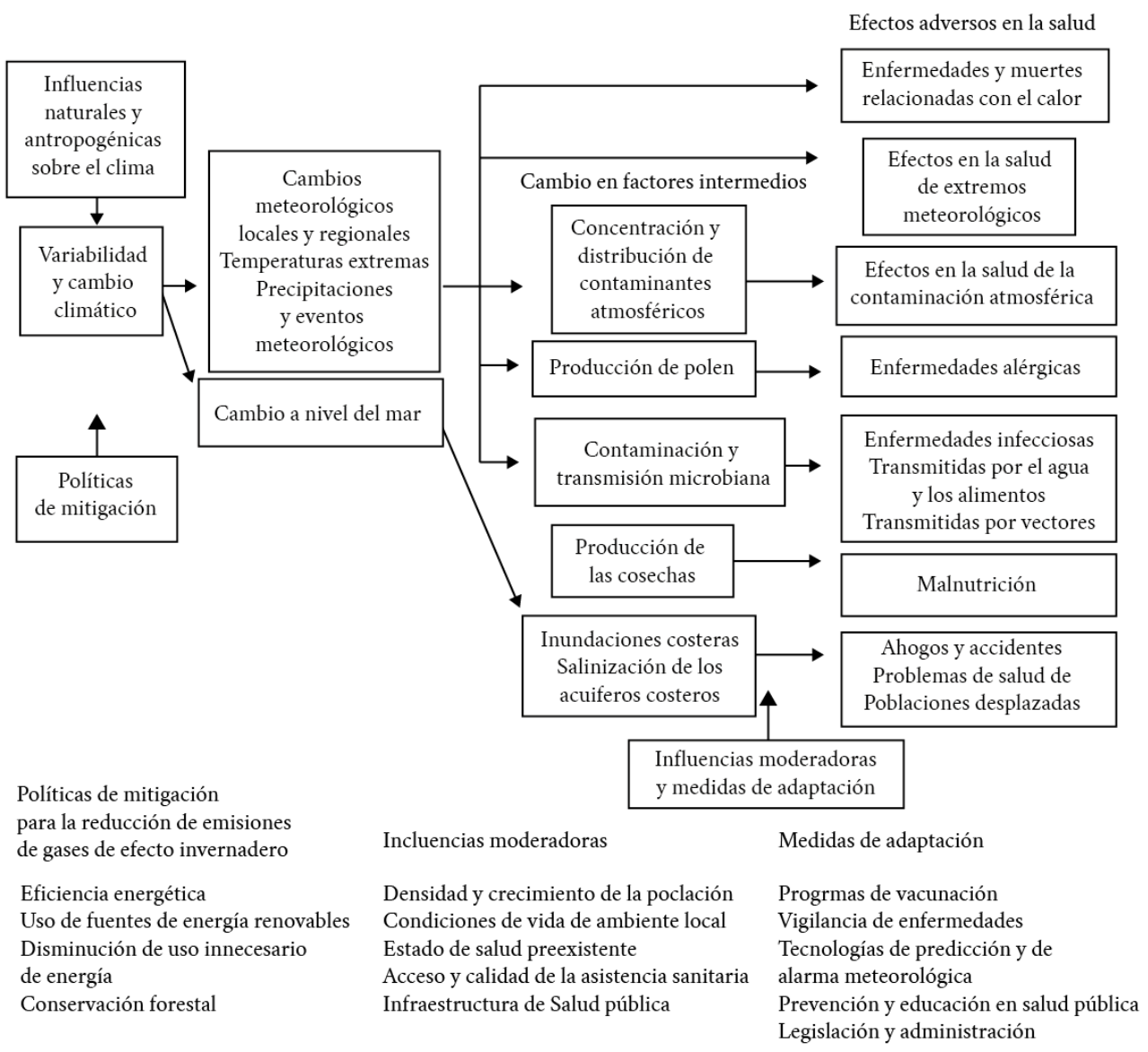

Figura 1. Efectos probables del cambio climático en la salud y sus posibles respuestas (adaptación, sistemas de vigilancia y prevención e investigación).

Fuente. Ballester F, Díaz J, Moreno J. Cambio climático y Salud Pública: escenarios después de la entrada en vigor del Protocolo de Kioto.

\section{Aumento de los efectos en la salud por cau- sa de los eventos climáticos extremos en El Salvador}

Según la Organización de las Naciones Unidas para la Alimentación y la Agricultura (FAO), El Salvador ha sufrido un incremento de temperatura en las últimas décadas de 1.3 grados centígrados, cuando el promedio global fue 0.8. En el futuro, la temperatura en el país podría subir 2.6 grados más. (FAO, 2017)
Con respecto a las enfermedades transmitidas por vectores, entre ellas zika, dengue y chikungunya, cuyos principales factores determinantes son: presencia del vector, condiciones climáticas (temperatura y precipitaciones), cambio climático - Fenómeno de El Niño, las epidemias en países vecinos, circulación de personas. La situación en El Salvador de acuerdo a datos del boletín epidemiológico de la semana 52 (23 al 29 de diciembre) de 2018, (MINSAL, 2019) es la siguiente:

Casos con sospecha de Zika de la semana 1 a 52 de 2018 se tuvo un acumulado de 481 casos con 
sospecha, lo cual significa un aumento discreto de 31 casos en la tendencia de casos, respecto del año 2017. Las edades más afectadas son menores de un año, seguido por el grupo de 20 a 29 años. Los departamentos con tasas más altas son: Chalatenango, San Vicente, Cabañas, La Paz, Ahuachapán y Santa Ana. (MINSAL, 2019)

Situación acumulada de Dengue, hasta la semana 50 de 2018 presenta un aumento del 197 \% con respecto al 2017, un aumento de las hospitalizaciones de 1244 en relación al año 2017. Las edades más afectadas son de 5 a 9 años, seguidos por los menores de un año y los de 1 a 4 años. Los departamentos con tasas más altas son Santa Ana, Chalatenango y Sonsonate (MINSAL, 2019).

Los casos de Chikungunya (hasta las semana 52 de 2018) han experimentado una importante reducción porcentual del $33 \%$ de casos sospechosos respecto a 2017. Los departamentos con tasas más altas son Chalatenango, San Vicente, Cuscatlán y Sonsonate. (MINSAL, 2019)

Las acciones realizadas para la eliminación de vectores son:

Inspecciones a viviendas, aplicación de bolsas de larvicida a criaderos de zancudos, aplicaciones de fumigación a viviendas, controles de foco, tratamientos de áreas colectivas, fumigaciones en áreas colectivas, perifoneo, distribución de material educativo, charlas y entrevistas educativas.

Incremento de la frecuencia de las enfermedades respiratorias debido a los cambios en la calidad del aire en El Salvador

Según la Organización Mundial de la Salud (OMS) San Salvador es considerado entre las ciudades y capitales de Latinoamérica con mayor nivel de contaminación en el aire, el material particular (PM) suspendido en el aire puede tener efectos adversos en la salud, produciendo problemas en los sistemas respiratorio y cardiovascular de la población. Por ejemplo, en el año 2013, Soyapango tuvo el promedio más alto de material particulado
PM 2.5 acumulado y tuvo 96 días con calidad del aire dañina. Ese mismo año hubo un aumento de las atenciones por asma no especificada, sumando 1165 consultas. (OMS/T21.2017)

San Salvador y Santa Tecla son las zonas más afectadas por contaminantes producidos por las emisiones de humo a partir del uso de diésel que están catalogados como contaminantes atmosféricos con efectos carcinógenos, mutágenos y tóxicos que son altamente nocivos para los seres humanos y tienen efectos graves en la salud.

Según el Ministerio de Salud de El Salvador (MINSAL, 2018) las principales fuentes de contaminación atmosférica son:

- Las Fuentes móviles: Autobuses transporte colectivo, microbuses (uso diésel), vehículos particulares.

- Actividades productivas: Industria, Agroindustria, Agrícola.

Según el Ministerio del Medio Ambiente y Recursos Naturales (MARN, 2016) el $60 \%$ de la contaminación atmosférica es producida por los automotores.

Según el Sistema Nacional de Vigilancia Epidemiológica «VigEpES», Los Casos de infección respiratoria aguda en 2018 fueron 1877786 (VigEpES, 2019).

\section{Aumento de la incidencia de las enferme- dades por la reducción en la disponibilidad y calidad del agua en El Salvador}

Según el Fondo Ambiental de El Salvador (FONAES, 2017) El Salvador tiene las condiciones ambientales más degradadas de América que afecta la calidad de vida de todos los salvadoreños. El país tiene la tasa de disponibilidad de agua dulce más baja per cápita en toda América Central, donde el $95 \%$ de los ríos presentan altos nivel de contaminación ambiental y la calidad del agua es mala, y solo un porcentaje muy pequeño aún es suscepti- 
ble de sostener vida. Adicionalmente, el cambio en el uso de la tierra (por ejemplo, la urbanización de las aguas de recarga acuífera) y la contaminación están causando una reducción en la disponibilidad del agua. La falta de acceso y mala calidad del agua afectan la calidad de vida, la productividad y la salud de la población y los ingresos de los diferentes sectores.

Según datos del Ministerio de Medio Ambiente y Recursos Naturales (MARN, 2016) en 2015 El Salvador tuvo reducciones de caudales de hasta el 95 \% en el Río Torola, el 70 \% en el Río Grande de San Miguel, el $95 \%$ en el Goascorán y entre el $60 \%$ y $80 \%$ en los ríos de la zona occidental y norte del país. Además, mostró una reducción en el nivel de todos los pozos entre 10 a 25 litros por segundo.

En El Salvador el agua se vuelve cada día más escasa debido al agotamiento de las zonas de recarga acuífera; la deforestación, la contaminación de las aguas superficiales y el agua subterránea; la pérdida de la capacidad de regulación e infiltración del agua; la creciente variabilidad climática, y el mal uso que se hace de este recurso natural. Además, la demanda hídrica está aumentando de forma constante, debido al crecimiento social y económico del país y deriva en una importante presión por sus usos, llegando a causar conflictos sociales y políticos.

Las zonas consideradas con estrés hídrico en El Salvador, según El Ministerio de Medio Ambiente y Recursos Naturales son Sonsonate, Usulután y San Vicente. (MARN, 2018)

Esto, sin duda impactará de manera significativa la salud de los salvadoreños ya que la escasez de agua afecta directa e indirectamente la salud, como enfermedades diarreicas, mala nutrición y desnutrición; por almacenamiento inadecuado y contaminación del agua o por escasez de alimentos, porque los cultivos son afectados.

En referencia a la enfermedad diarreica aguda en El Salvador, la tasa acumulada hasta la semana 52 del 2018 fue de 5244 casos x 100mil/habitantes, al compararla con el año 2017 presenta un incremen- to de riesgo de 267 casos x $100 \mathrm{mil} /$ habitantes. El $51 \%$ de los egresos por diarrea corresponde a hombres, la mayor proporción de egresos se encuentra en menores de 5 años (69\%) seguido por los adultos mayores de 59 años (7\%). (VigEpES, 2019)

Debido a la dificultad hídrica en El Salvador, el MARN con el apoyo del Gobierno de España, a través del Fondo de Agua, elaboró el Plan Nacional Hídrico que orienta, ordena y prioriza los usos de agua. Este plan contempla medidas en cuatro ejes temáticos: «Aprovechamiento sostenible, calidad del agua, gestión de riesgos por fenómenos extremos y gobernanza; parte de sus objetivos principales es la caracterización de los problemas actuales y previsibles relacionados con el agua y la propuesta de solución». (MARN, 2016)

El Salvador cuenta con la política de cambio climático que plantea el mejor manejo del agua, acciones para buscar la recuperación del bosque, así la tierra podrá retener más agua. Además, dentro de la política se prevén acciones como transferir conocimiento a los productores sobre la protección de los cuerpos de agua (estos no siempre manejan la información de hasta qué punto es importante la conservación del recurso), prácticas de adaptación como un manejo distinto del suelo, obras de ingeniería para proteger y cosechar humedad o agua y enseñar a usar ciertos materiales vegetales para impedir la erosión.

Es evidente que el cambio climático tiene incidencia social y hay necesidad de políticas públicas, ya que afecta la sociedad, su hábitat, su salud y la economía, siendo los países en vías de desarrollo los más afectados y de los estratos sociales aquellos que viven en extrema pobreza.

En referencia a «el cambio climático (CC), los movimientos sociales (MS) y las políticas públicas (PP) ha sido bastante prolífica en cada uno de dichos campos tomados por separado, pero débil o incipiente en sus interconexiones» (Bravo F., 2014), esto significa que el problema del cambio climático y sus efectos debe ser manejado multidisciplinariamente para obtener mejores resultados. 
Hay que estar conscientes que para contrarrestar los efectos del cambio climático, las soluciones deben ser orientadas a un mayor involucramiento de todos los sectores sociales, «Las soluciones a largo plazo se basan en la capacidad de construir sociedades más justas, equitativas, con un sentido de solidaridad que active la participación y la corresponsabilidad social.». (Soares, D, \&Murillo-Licea, 2013)

\section{¿Qué medidas deben aplicarse para dismi- nuir la contaminación atmosférica?}

Entre las medidas más importantes tenemos las medidas legislativas en las cuales hay que hacer normas de calidad, pero velar por que estas sean cumplidas a totalidad y sin excepción, además de realizar acciones por medio de la planificación de las comunidades urbanas y rurales para tratar a la vez de detectar y reducir los diferentes contaminantes del aire y así poder controlar la fuente de origen todo esto por medio de un sistema de vigilancia sistémico y evaluado.

La contaminación del aire representa un importante riesgo medioambiental para la salud; sin embargo, disminuyendo los niveles de contaminación del aire, El Salvador puede reducir la carga de morbilidad. Será necesario el monitoreo, determinar el grado de contaminación del aire ambiental y su relación con las condiciones de la exposición, riesgos a la salud, como efectos adversos. Precisar la contribución de las diversas fuentes a la contaminación de la atmósfera, también evaluar los resultados de las medidas de prevención y control y, en particular, la aplicación de las normas sobre calidad del aire.

Además, se debe promover entre la población general la conciencia sobre los efectos de los contaminantes, las causas que los originan y la importancia de colaborar y participar en los esfuerzos por conservar el recurso aire.

Los vehículos automotores son la principal fuente de contaminación del aire en San Salvador, el Estado Salvadoreño por medio de sus institucio- nes (Ministerio de Medio Ambiente y Recursos Naturales, Ministerio de Salud, Vice Ministerio de Transporte) es responsable de garantizar la salud de la población, por lo que tiene el deber de dar cumplimiento a la regulación de las emisiones de gases de los vehículos automotores.

\section{¿Cómo debemos responder a la reducción en la disponibilidad y calidad del agua en El Salvador?}

Enfrentar la problemática salvadoreña, sobre la baja cobertura del servicio de agua, implica diseñar nuevas formas de gestión descentralizadas del agua, nuevas formas de participación de las organizaciones e instituciones públicas y privadas en el ámbito territorial; implica mejorar la captación de recursos hídricos, a través de la gestión de cuencas; así también la orientación de recursos económicos, materiales y humanos para la conservación de las cuencas; pasa por la inversión en infraestructura que permita ampliar la red de servicios y las mejoras institucionales en la prestación del servicio; es necesario adecuar y actualizar las tarifas, modificar radicalmente el actual sistema de subsidio y focalizarlo a los más pobres y necesitados; requiere de mayor conciencia y responsabilidad ambiental.

Se deben realizar inspecciones y vigilancias sanitarias del agua para prevención y control de condiciones potencialmente peligrosas, incluye epidemias de enfermedades transmitidas por el agua, ya que esta consiste en la evaluación y supervisión permanente y alerta del abastecimiento.

Además, propiciar una ley en donde se implique atender la demanda del agua y, al mismo tiempo, proteger las aguas superficiales como subterráneas para que se alcance un buen estado. Diseñar una estrategia de ampliación de cobertura de agua potable y saneamiento.

También fomentar en la población el ahorro del agua que nos asegure que se desenvuelva al medioambiente con calidad adecuada y al mismo tiempo garantiza el suministro de la demanda, 
mediante fuentes alternativas de agua. Además, descentralizar el sistema de agua potable y saneamiento en El Salvador.

Así mismo, fomentar una agricultura eficiente, en donde se enseñe a los agricultores a ajustar el agua que hay que aportar a los cultivos en los riegos, además de enseñarles a mejorar sistemas de sombra para reducir la temperatura y con ello la evaporación y conservar la humedad del suelo.

Enseñar a la población a reutilizar el agua, cuyo beneficio es la posibilidad de reservar el agua de mejor calidad para los usos más exigentes, tales como la producción de agua potable.

\section{Conclusión}

El cambio climático trae repercusiones en la salud de la población que influye en el desarrollo de enfermedades infecciosas y las enfermedades no infecciosas, principalmente, enfermedades cardiorrespiratorias, así como aumento de las enfermedades causadas por vectores. El cambio climático contribuye progresivamente a la carga global de enfermedades y muertes prematuras.

Los efectos que ha provocado el cambio climático son aumento de la mortalidad por temperaturas extremas, incremento de enfermedades por contaminación ambiental, malnutrición debido a las sequías y al desequilibrio estacional de las cosechas, aumento de muertes, enfermedades y daños debido a inundaciones, tormentas, incendios y sequías.

Las acciones para contrarrestar los efectos del cambio climático deben tener un mayor involucramiento de todos los sectores sociales; como gobierno, instituciones educativas públicas y privadas, empresas, industrias, maquilas, entre otros; fortaleciendo la coordinación interinstitucional e intersectorial, mejorando la prevención y preparación ante los cambios del clima, mitigación de los desastres y reduciendo la vulnerabilidad.
El desafío ambiental más grave para la salud es el cambio climático, por lo cual es importante informar a la población sobre las potenciales consecuencias en salud y de esta manera crear conciencia sobre el mismo, lo que posibilitará implementar acciones para contrarrestar dicho impacto (por ejemplo, disminuir de manera importante las emisiones de carbono y otros agentes que contribuyen al cambio climático) así como omitir otras que aceleran la degradación ambiental.

Dado que las repercusiones del cambio climático nos afectan a todos, lo que se debe requerir de la población es un cambio de comportamiento, una conducta proambiental, un cambio de estilo de vida. Se trata de cambiar nuestros conceptos cognitivamente, pero también conservar y proteger el entorno, no sobrexplotar los recursos, disminuir el consumo de energía eléctrica, evitar la contaminación, por otra parte, conectar nuestras actividades equilibradamente con los procesos ambientales en curso.

En torno al tema de la participación ciudadana se encuentra un problema actitudinal. Sus nexos culturales dominantes siguen estando bajo la sombra del paternalismo y asistencialismo. Se puede contribuir al cambio actitudinal, creando las condiciones para un ejercicio saludable de participación entre los agentes involucrados.

Una de las mayores debilidades ha sido la falta de consulta y consenso de las diferentes iniciativas, para obtener los objetivos que cada una de ellas contemplaba, es decir la dispersión y no articulación con la que trabajan los diferentes actores que intervienen en su gestión, con una insuficiente coordinación. La poca o nula participación ciudadana en la generación de propuestas de mejora en las iniciativas, dificultan que la población se involucre, sea parte o haga para sí, la defensa de una propuesta en particular.

La ausencia de Políticas de Estado, en materia de seguridad social llámese, salud, saneamiento básico, provisión de agua apta para consumo hu- 
mano, conectividad y seguridad, ha dificultado el desarrollo del país en materia social. Las políticas se implementan como Planes Quinquenales que coinciden con los gobiernos en turno, situación que no permite la continuidad en aspectos positivos o el aprendizaje basado en aspectos negativos.

Aunque El Salvador ha realizado avances en respuesta al impacto del cambio climático en salud dista mucho de lograrlo, por lo que se requiere seguir trabajando en esto, como diseñar estrategias de financiamiento para dar sostenibilidad y sustentabilidad en el tiempo por medio de impuestos graduales y consecutivos y con el involucramiento de diversos sectores. Es decir, asegurar mecanis- mos de financiamiento y de asignación de recursos que sean solidarios y eficientes, impulsar, profundizar y defender una genuina y amplia participación social y comunitaria en salud y medioambiente, realizar investigación y evaluación del impacto en salud del cambio climático en El Salvador, fomentar una educación proambiental desde los primeros grados hasta la educación superior en todas las carreras.

En síntesis las respuestas para reducir el impacto del cambio climático en la salud deben ser enfocadas en legislación, acciones técnicas, alerta, educación, cultura y conducta. 


\section{Fuentes de información consultadas}

1. Álvarez Brito, Arnaldo F. (2013) «La biotecnología vegetal: ¿Una alternativa para el enfrentamiento a los impactos del cambio climático en Cuba? (Spanish)». The plant biotechnology: An alternative for the confrontation to the impacts of the climatic change in Cuba? (English) 18, no. 5, Instituto de Investigaciones Agro-Forestales. Instituto de Investigaciones Agro-Forestales. La Habana, Cuba,: 195-201. Disponible en: (http://search. ebscohost.com/login.aspx?direct $=$ true $\& \mathrm{db}=\mathrm{zb}$ $\underline{\text { h\&AN}=95274779 \& \text { lang }=\text { es\&site }=\text { ehost-live }}$

2. Ballester F, Díaz J, Moreno J. (2006). Cambio climático y Salud Pública: escenarios después de la entrada en vigor del Protocolo de Kioto. Gac Sanit; 20 (supl 1):160-74.

3. Ballester, Ferran. (2005) «Contaminación Atmosférica, Cambio Climático y Salud». Revista Española de Salud Pública, vol. 79, núm. 2.Madrid, España. pp. 159-175. Disponible en: http://www. redalyc.org/articulo.oa?id=17079207.

4. Bravo Alarcón, Fernando. (2014) «Cambio climático, movimientos sociales y políticas públicas. Una vinculación necesaria». Debates en Sociología 39: 181-85. Disponible en: http:// search.ebscohost.com/login.aspx?direct $=$ true\& $\underline{\mathrm{db}=\mathrm{a} 9 \mathrm{~h} \& \mathrm{AN}=111379835 \text { \&lang }=\text { es \& } \mathrm{site}=\text { eho }}$ st-live.

5. de la Cuadra, Fernando. (2013), «Julio Postigo (editor), Cambio climático, movimientos sociales y políticas públicas. Una vinculación necesaria, CLACSO/ICAL, Santiago, 302 p.» Polis. Revista Latinoamericana, no. 36. Disponible en: https:// polis.revues.org/9651.
6. Espejel Rodríguez, A, y Flores Hernández, A. (septiembre de 2015) «Conocimiento Y Percepción Del Calentamiento Global En Jóvenes Del Bachillerato, Tlaxcala». Revista mexicana de ciencias agrícolas 6, no. 6: 127790. Disponible en: http://www.scielo.org.mx/ scielo.php? script $=$ sci abstract $\&$ pid $=\mathrm{S} 2007$ 09342015000600011\&lng=es\&nrm=iso\&tlng $=$ es.

7. FAO (2017). «Cronología del Corredor Seco: El acelerador de la resiliencia en Centroamérica | Agronoticias: Actualidad agropecuaria de América Latina y el Caribe | Organización de las Naciones Unidas para la Alimentación y la Agricultura». Accedido 31 de enero de 2019. Disponible en: http://www.fao.org/in-action/ agronoticias/detail/es/c/1024540/.

8. FONAES, (2017), «Recurso Hídrico / Fondo Ambiental de El Salvador». Accedido 31 de enero de 2019. Disponible en: http://fonaes.gob.sv/index. php/temas/recurso-hidrico/.

9. González, Y, Fernández, Y, \& Gutiérrez, T. (2013) «El Cambio Climático y sus efectos en la salud». Climate change and its effects on health. 51, no 3 : 331-37. Disponible en: http://search.ebscohost. $\underline{\text { com } / \operatorname{login} . \text { aspx }}$ ?direct $=$ true $\& \mathrm{db}=\mathrm{a} 9 \mathrm{~h} \& \mathrm{AN}=9493$ 8820\&lang $=$ es\&site $=$ ehost-live.

10. Gunther, María G. (enero de 2014) «Diálogo En Construcción: Cambio Climático, Movimientos Sociales Y Políticas Públicas». Política y Cultura, n.o 41: 229-32. Disponible en: http://search. ebscohost.com/login.aspx?direct $=$ true $\& \mathrm{db}=\mathrm{a} 9$ $\underline{\mathrm{h} \& \mathrm{AN}=96883588 \text { \&lang }=\text { es \&site=ehost-live. }}$

11. Intergovernmental Panel on Climate Change (IPCC), (2001). Houghton J, Ding J, Griggs D, Noger M, et al. Climate Change 2001. The Scientific Basis. New York: Cambridge University Press. 
12. Intergovernmental Panel on Climate Change (IPCC). (1996). Houghton J. Ed. Climate Change 1995: The Science of Climate Change. Cambridge, UK: Cambridge University Press.

13. International Panel for Climate Change (IPCC). (2001), Climate Change 2001. En: Houghton JT, Ding J, Griggs DJ, Noguer M, Vander Linden PJ, Xiaosu D, editors. The Scientific Basis. Cambridge University Press: Cambridge.

14. Kenney W.L, Hodgson J.L. (1987). Heat tolerance, thermoregulation and ageing. Sports Med.; 4:44656.

15. Kuhn K, Campbell-Lendrum D, Haines A, Cox J, Corvalán C, \& Anker M. (2005) Using Climate to predict infectious disease epidemics. WHO. Págs.147.

16. Kunst A.E, Looman C.W, Mackenbach J.P. (1993). Outdoor air temperature and mortality in the Netherlands: a time series analysis. Am J Epidemiol.;1137:331-41

17. Martínez, J. T. (J. C. Postigo, Ed.), (2013), «Cambio climático, movimientos sociales y políticas públicas: Una vinculación necesaria». (1 $1^{\text {a. }}$ ed.). CLACSO.ICAL.INTE-PUCP. Santiago de Chile, p.239.

18. Ministerio de Medio Ambiente y Recursos Naturales. | MARN |, (2016) «Gobierno decreta emergencia y alerta nacional para atender desabastecimiento de agua». Accedido 8 de septiembre de 2016. Disponible en: http://www. marn.gob.sv/gobierno-decreta-emergencia-yalerta-nacional-para-atender-desabastecimiento-de-agua/.

19. MINSAL (2019). «Dirección de Vigilancia Sanitaria». MINSAL (blog). Accedido 31 de enero de 2019. Disponible en: http://www.salud.gob. sv/direccion-de-vigilancia-sanitaria/.
20. MINSAL. (2019). «Boletines Epidemiológicos 2019». MINSAL (blog). Accedido 31 de enero de 2019. Disponible en: http://www.salud.gob.sv/ boletines-epidemiologicos-2019/.

21. Ochoa, M, Rosa Castellanos, R, Ochoa Z, \& Oliveros,J. (julio de 2015). «Variabilidad Y Cambio Climáticos: Su repercusión en la salud». Variability and climatic changes: their influence on health. 19, n.o 7: 870-82. Disponible en: http://search.ebscohost.com/login.aspx?direct $=\mathrm{t}$ $\underline{\text { rue } \& \mathrm{db}=\mathrm{a} 9 \mathrm{~h} \& \mathrm{AN}=108519820 \& \text { lang }=\mathrm{es} \& \text { site }=\mathrm{e}}$ host-live.

22. OMS (2016). «Cambio climático y salud». WHO. Accedido 8 de septiembre de 2016. Disponible en:http://www.who.int/mediacentre/factsheets/ fs266/es/.

23. OMS/T21. (2017). «La OMS alerta de la contaminación ambiental». Tendencias 21. Ciencia, tecnología, sociedad y cultura. Accedido 25 de enero de 2019. Disponible en: https://www. tendencias21.net/La-OMS-alerta-de-la-contaminacion-ambiental_a44531.html.

24. Organización Mundial de la Salud (1948). Constitución de la Organización Mundial de la Salud [Documento en línea]. Disponible en: http:// www.who.

25. Patz J.A, Kovats R.S. (2002). Hotspots in climate change and human health. BMJ; 325:1094-8.

26. Presidencia de la República de El Salvador. (2016). «Gobierno presenta el primer Plan Nacional del Cambio Climático». Accedido 8 de septiembre de 2016. Disponible en: http://www.presidencia. gob.sv.

27. Rozas O, Germán. (2013). «Aproximación Psico Comunitario Ambiental Al Problema De Calentamiento Global». Revista de Psicología, vol. XII, núm. 2. Santiago, Chile 2003. pp. 19-34. Disponible en: http://www.redalyc.org/articulo. oa?id=26412203. 
28. Soares, D., \& Murillo-Licea, D. (2013). Gestión de riesgo de desastres, género y cambio climático. Percepciones sociales en Yucatán, México. Cuadernos de Desarrollo Rural, 10 (72), 181-199.

29. Sutherst R. (2001). Global change and human vulnerability to Vector-Born diseases. Clin Microbiol Rev; 5:136-73.
30. OMS (2017). «WHO Household energy database». WHO. Accedido 25 de enero de 2019. Disponible en: http://www.who.int/airpollution/data/household-energy-database/en/.

31. Woodward A, Hales S, Litidamu N, Phillips D, Martin J. (2000). Protecting human health in a changing world: the role of social and economic development. Bull World Health Organ;78(9):1148-55. 